\title{
Diversity-optimized cooperation on complex networks
}

\author{
Han-Xin Yang, ${ }^{1}$ Wen-Xu Wang, ${ }^{2}$ Zhi-Xi Wu, ${ }^{3}$ Ying-Cheng Lai, ${ }^{2,4}$ and Bing-Hong Wang ${ }^{1,5}$ \\ ${ }^{1}$ Department of Modern Physics, University of Science and Technology of China, Hefei 230026, China \\ ${ }^{2}$ Department of Electrical Engineering, Arizona State University, Tempe, Arizona 85287, USA \\ ${ }^{3}$ Department of Physics, Umeå University, 90187 Umeå, Sweden \\ ${ }^{4}$ Department of Physics, Arizona State University, Tempe, Arizona 85287, USA \\ ${ }^{5}$ The Research Center for Complex System Science, University of Shanghai for Science and Technology, Shanghai 200093, China
}

(Received 11 December 2008; published 19 May 2009)

\begin{abstract}
We propose a strategy for achieving maximum cooperation in evolutionary games on complex networks. Each individual is assigned a weight that is proportional to the power of its degree, where the exponent $\alpha$ is an adjustable parameter that controls the level of diversity among individuals in the network. During the evolution, every individual chooses one of its neighbors as a reference with a probability proportional to the weight of the neighbor, and updates its strategy depending on their payoff difference. It is found that there exists an optimal value of $\alpha$, for which the level of cooperation reaches maximum. This phenomenon indicates that, although high-degree individuals play a prominent role in maintaining the cooperation, too strong influences from the hubs may counterintuitively inhibit the diffusion of cooperation. Other pertinent quantities such as the payoff, the cooperator density as a function of the degree, and the payoff distribution are also investigated computationally and theoretically. Our results suggest that in order to achieve strong cooperation on a complex network, individuals should learn more frequently from neighbors with higher degrees, but only to a certain extent.
\end{abstract}

DOI: 10.1103/PhysRevE.79.056107

PACS number(s): 89.65.-s, 02.50.Le, 07.05.Tp, 87.23.Kg

\section{INTRODUCTION}

Cooperation is ubiquitous in a variety of complex systems ranging from natural to economical and social systems [1]. However, understanding the emergence and persistence of cooperative behaviors remains a challenge. In this regard, evolutionary game theory has provided a suitable mathematical framework to address this fundamental phenomenon in complex systems $[2,3]$. For example, games such as the prisoner's dilemma game (PDG) and the snowdrift game (SG) as a paradigm to explain cooperative behaviors through pairwise interactions [4] have been studied [5]. A more general model for cooperation among social species, including human behaviors ranging from family relations to global warming [6], is the public goods game (PGG) that allows more general group interactions. In a typical PGG played by $N$ individuals, each individual can choose to cooperate or defect. Cooperators, denoted by $C$, contribute an amount $c$ to the PGG, while defectors $(D)$ do not contribute. The total contribution is multiplied by a factor $r$, and is then redistributed uniformly among all players. Similar to the situation associated with PDG and SG, defection represents the dominant strategy leading to the deterioration of cooperation. This somewhat generates a dilemma, as cooperation has been widely observed in all kinds of complex systems.

To resolve the dilemma associated with the PGG, extensive research has been carried out in the past few years [7-13]. In Ref. [7], voluntary participation is introduced into the PGG, which results in a substantial and persistent willingness to cooperate. In Ref. [8], voluntary participation in PGG on a square lattice is studied with the result that the introduction of loners who refuse to participate and rather rely on some small but fixed income leads to a cyclic dominance of strategies and promotes substantial levels of coop- eration. The effects of inhomogeneous activity in the PGG have been studied in Ref. [12], where the cooperation level is found to be considerably enhanced. Quite recently, social diversity has been introduced by means of heterogeneous graphs [13]. It is found that diversity associated with the number and the size of the PGG, where each individual participates and contributes to each game, can promote strong cooperation. This finding is quite surprising as that cooperation can be remarkably facilitated in the absence of other mechanisms based on, for example, reputation and punishment [14-16].

In this paper, we investigate, quantitatively, the effects of individual diversity on the emergence and the evolution of cooperation in the framework of evolutionary games. Our main motivation comes from the realization that, while diversity can promote cooperation, too much diversity will not be desirable for cooperation. There must then exist an optimal level of diversity for which the degree of cooperation is maximized. An analogous situation occurs in physics: stochastic resonance [17], where a proper level of noise can result in better system response to, for example, weak periodic signals, but too much noise will certainly not be beneficial. An approach to addressing diversity is to consider PGG on complex systems consisting of heterogeneous individuals [13]. For convenience, we choose scale-free networks that typically exhibit a high level of heterogeneity, e.g., in the degree distribution. To incorporate heterogeneity in the PGG, we introduce diversity in the strategy updating according to the degrees of the individuals (nodes) in the network. This is reasonable because updating rules play an important role in the evolution of cooperation, where individuals can update their strategies by adopting different rules in the evolutionary game. For example, in [18], a stochastic evolutionary rule is proposed: when updating strategy, each individual randomly selects one of its neighbors to refer to, and adopts the strat- 
egy of the reference with a probability determined by their payoff difference.

Due to the existence of social diversity [19], the impact of different individuals can be different. For example, leaders may have stronger influence than others and may be followed more frequently. That is, different neighbors can have different impacts on one's behavior. As a result, the selection of a neighbor as a reference by an individual is usually not completely random in the decision-making process [20-22]. Our approach is then as follows. We incorporate the heterogeneous network topology in strategy updating by assuming a preferential imitation rule so that the evolution of cooperative behavior in the PGG can be addressed in a quantitative manner. Specifically, each individual $i$ is assigned a weight $k_{i}^{\alpha}$, where $k_{i}$ is the degree of the individual and $\alpha$ is an adjustable parameter which we call the diversity parameter. During the evolutionary process, every individual chooses one of its neighbors as a reference with a probability proportional to the neighbor's weight. We find that, in general, there exists an optimal value of $\alpha$ that leads to the highest level of cooperation, in agreement with physical intuition. To place the finding on a solid foundation, we work out a physical theory, aided by numerical computations, to explain the existence of the optimal cooperation. Relevant phenomena such as the payoff as a function of node degree and the wealth distribution are also investigated. An encouraging result is that our analysis naturally yields a power-law wealth distribution but with an exponential start-off, which has been observed in real-world complex systems.

In Sec. II, we introduce our strategy updating rule in PGGs on scale-free networks and present numerical observations for the resonancelike phenomenon of the emergence of optimal cooperation. In Sec. III, a physical theory is presented to explain the phenomenon. Discussions are presented in Sec. IV.

\section{MODEL AND NUMERICAL EVIDENCE FOR OPTIMAL COOPERATION}

According to Ref. [13], each individual $i$ participates in interactions in $k_{i}+1$ neighborhoods that center about $i$ and its $k_{i}$ neighbors, where each neighborhood contains a central node and all nodes that are directly connected to it. Each cooperator contributes a total $\operatorname{cost} c=1$ shared equally among all the neighborhoods that it engages. The strategy is $s_{x}=1$ if a $C$ game is played and $s_{x}=0$ if a $D$ game is played. The payoff of an individual $x$ with strategy $s_{x}$ associated with the neighborhood centered at an individual $y$ is given by

$$
m_{x, y}=\frac{r}{k_{y}+1} \sum_{i=0}^{k_{y}} \frac{s_{i}}{k_{i}+1}-\frac{s_{x}}{k_{x+1}},
$$

where $i=0$ stands for $y, s_{i}$ is the strategy of the neighbor $i$ of $y$, and $k_{i}$ is its degree. The total payoff of player $x$ is

$$
M_{x}=\sum_{y \in \Omega_{x}} m_{x, y},
$$

where $\Omega_{x}$ denotes the community of neighbors of $x$ and itself. In our model, each individual $i$ is assigned a weight $k_{i}^{\alpha}$.
During the evolution, each individual chooses one of its neighbors as a reference with a probability proportional to the neighbor's weight, i.e., the probability $A_{x \rightarrow y}$ of $x$ selecting a neighbor $y$ is

$$
R_{x \rightarrow y}=\frac{k_{y}^{\alpha}}{\sum_{j} k_{j}^{\alpha}},
$$

where the sum is over all neighbors of $x$. For $\alpha>0(<0)$, high-degree (low-degree) individuals have larger probabilities to be selected as references. In the case of $\alpha=0$, individuals are chosen randomly and uniformly as references for a neighbor. Our weighted selection rule is related with those in Refs. [23,24]. In particular, in Ref. [23], the player has a specific strategy-passing capacity, and reproduction restriction in the PD game has been studied in Ref. [24]. After a neighbor $y$ is selected, player $x$ adopts $y$ 's strategy $s_{y}$ with the probability:

$$
W\left(s_{x} \rightarrow s_{y}\right)=\frac{1}{1+\exp \left[\left(M_{x}-M_{y}\right) / \kappa\right]},
$$

where $\kappa$ denotes the amplitude of noise. Following previous studies $[19,25-28]$, we set $\kappa=0.1$.

To incorporate diversity in our model, we assume that players are nodes in a standard scale-free network [29]. In the network model, there are $m_{0}$ nodes initially. At each time step, a new node with $m$ edges is added and preferentially attached to $m$ existing nodes with probabilities proportional to the degrees of existing nodes. The average connectivity of the network is controlled by $m$, i.e., $\langle k\rangle=2 m$, and the degree distribution is power law with the exponent -3 . In all simulations, the population is set to be $N=2000$ and the average connectivity is $\langle k\rangle=4$. Initially, the two strategies, $C$ and $D$, are randomly distributed among the individuals with equal probability $1 / 2$. An indicator of the final degree of cooperation is the cooperator density $\rho_{c}$ in the steady state, which is the fraction of the population involved in cooperation. In all simulations, $\rho_{c}$ is obtained by averaging over the last 2000 Monte Carlo time steps from a total of 30000 steps. Each data point results from 20 different network realizations with 20 runs for each realization.

Figure 1 shows $\rho_{c}$ as a function of the multiplication factor $r(\geq 1)$ for different values of $\alpha$. It can be seen that, for fixed $r$, different values of $\alpha$ can affect the final cooperation level dramatically. For $\alpha \leq 0, \rho_{c}$ vanishes below some threshold values of $r$. For $\alpha>0$, cooperation can survive even for $r=1$. To characterize the effect of varying $\alpha$ on cooperation, we plot $\rho_{c}$ as a function of the diversity parameter $\alpha$ for different values of $r$, as shown in Fig. 2. The phenomenon of optimal cooperation is unequivocal: for fixed value of $r$, there exists an optimal value of $\alpha$ for which $\rho_{c}$ is maximized.

We also observe that the payoffs of players can be quite different due to the difference in the number of groups that they participate in. This leads to a biased effect of noise $\kappa$ on the strategy updating as determined by Eq. (4). Thus, for nodes with different degrees, a fixed value of $\kappa$ effectively corresponds to different noise amplitudes. To eliminate the bias of noise effect on the strategy updating and to test the robustness of the phenomenon of optimal cooperation, we 


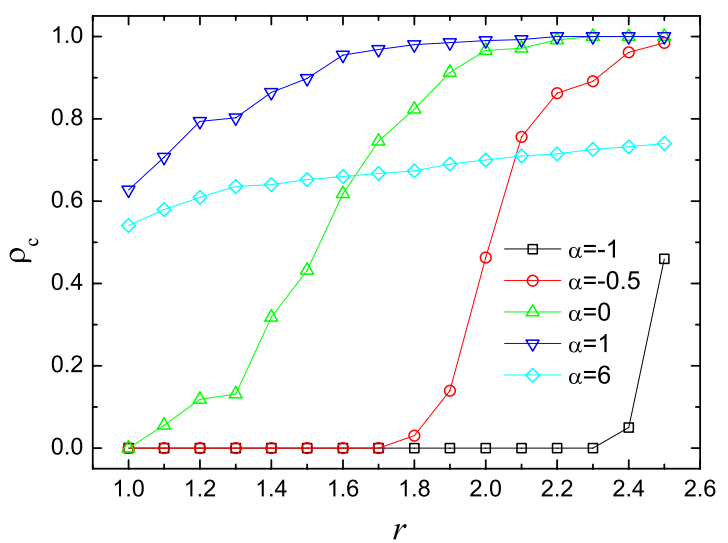

FIG. 1. (Color online) Steady-state cooperator density $\rho_{c}$ versus the multiplication factor $r$ for different values of $\alpha$.

study the dependence of $\rho_{c}$ on $\alpha$ by adopting normalized payoffs in Eq. (4). In particular, an arbitrary individual $i$ 's payoff is normalized by $k_{i}+1$, the number of groups that $i$ belongs to. Simulation results are shown in the inset of Fig. 2. We see that $\rho_{c}$ still peaks at some specific value of $\alpha$ and the phenomenon of the occurrence of optimal cooperation is robust.

\section{PAYOFF PROPERTIES AND OPTIMAL COOPERATION}

In the emergence of cooperation in PGGs, the standard approach is the mean-field theory. The main result in the literature [30-32] is that, for a well-mixed population to which the mean-field approximation is applicable, cooperation eventually dominates for $r>N$ for random mixture of initial conditions (i.e., equal initial probabilities of playing $C$ and $D$ ). The opposite occurs for $r<N$. These results, however, do not explain the optimal-cooperation phenomenon as exemplified in Fig. 2.

\section{A. Relation between individual degree and payoff}

To explain Fig. 2, we first investigate the role of hub nodes by computing the payoffs that hubs gain. It has been

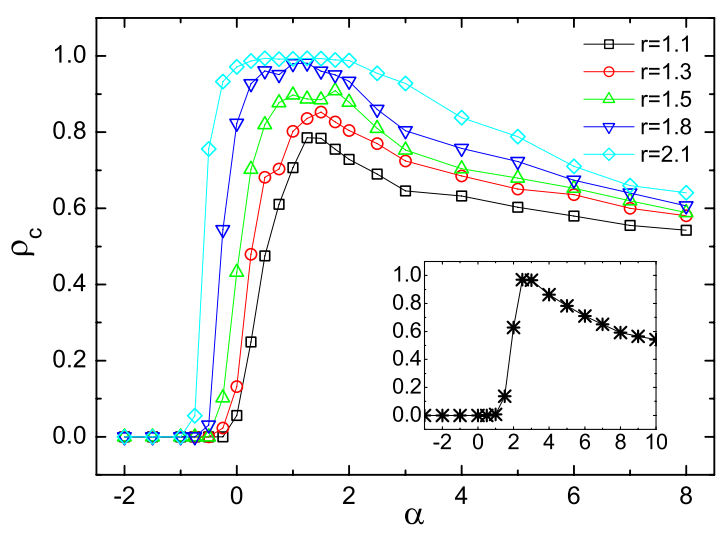

FIG. 2. (Color online) Steady-state cooperator density $\rho_{c}$ as a function of $\alpha$ for different values of $r$. Apparently $\rho_{c}$ can be optimized by the diversity parameter $\alpha$. The inset is $\rho_{c}$ versus $\alpha$ for $r$ $=3.5$ for the case where normalized payoffs are used.

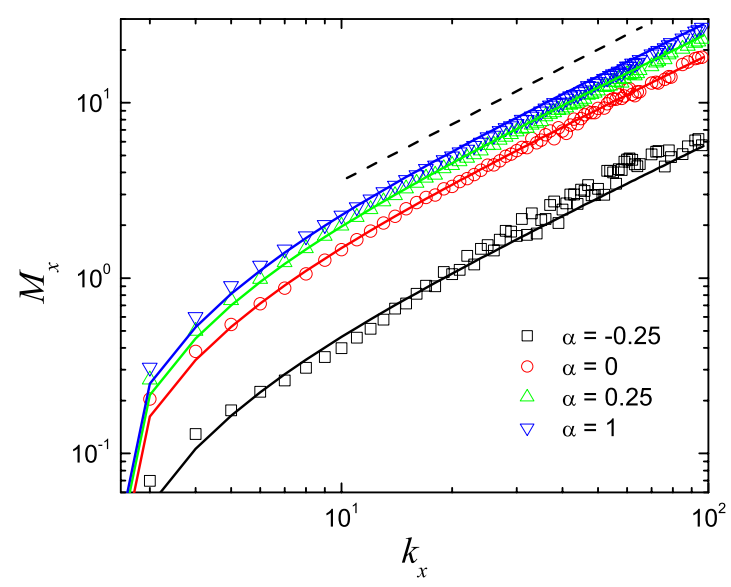

FIG. 3. (Color online) Payoff as a function of degree for different values of $\alpha$ when the system's cooperation level is stable. The multiplication factor is $r=1.6$. The solid curves are theoretical results from Eq. (9). The dashed line is of unit slope.

pointed out that in a scale-free network, the hubs (highdegree individuals) play a prominent role in maintaining the cooperation [13,33,34]. As shown in Fig. 3, for high-degree individuals, payoffs are apparently linearly correlated with their degrees, but for low-degree nodes, deviations from the linear behavior occur. These behaviors can in fact be explained in the framework of mean-field theory. In particular, assuming that the frequency of cooperation is given by $\rho_{c}$, we can express the payoff $m_{x, y}$ as

$$
m_{x, y}=\frac{r}{k_{y}+1}\left(\sum_{i=1}^{N} A_{i y} \frac{\rho_{c}}{k_{i}+1}+\frac{\rho_{c}}{k_{y}+1}\right)-\frac{\rho_{c}}{k_{x}+1},
$$

where $A_{i y}$ is the adjacency matrix of the underlying network. Neglecting the degree-degree correlation, we have

$$
\begin{aligned}
\sum_{i=1}^{N} A_{i y} \frac{1}{k_{i}+1} & =k_{y} \sum_{k_{\min }}^{k_{\max }} P\left(k^{\prime} \mid k_{y}\right) \frac{1}{k^{\prime}+1} \\
& =k_{y} \sum_{k_{\min }}^{k_{\max }} \frac{k^{\prime} P\left(k^{\prime}\right)}{\langle k\rangle} \frac{1}{k^{\prime}+1} \\
& =k_{y}\left\langle\frac{k}{k+1}\right\rangle\langle k\rangle,
\end{aligned}
$$

where $\langle\cdot\rangle$ denotes the average of all nodes, $P\left(k^{\prime}\right)$ is the degree distribution of network nodes, $P\left(k^{\prime} \mid k_{y}\right)$ is the joint degree distribution defined by $P\left(k^{\prime} \mid k_{y}\right)=k^{\prime} P\left(k^{\prime}\right) /\langle k\rangle$, and the identity

$$
\sum_{k_{\min }}^{k_{\max }} P\left(k^{\prime}\right) \frac{k^{\prime}}{k^{\prime}+1}=\left\langle\frac{k}{k+1}\right\rangle
$$

has been used. Substituting Eq. (6) in Eq. (5), we obtain

$$
m_{x, y}=\rho_{c}\left[\frac{r}{\langle k\rangle}\left\langle\frac{k}{k+1}\right\rangle \frac{k_{y}}{k_{y}+1}+\frac{r}{\left(k_{y}+1\right)^{2}}-\frac{1}{k_{x}+1}\right] .
$$

The total payoff is then given by 


$$
\begin{aligned}
M_{x}= & \sum_{y=1}^{N} A_{x y} m_{x, y}+m_{x, x} \\
= & \rho_{c}\left[\frac{r\left\langle\frac{k}{k+1}\right\rangle^{N}}{\langle k\rangle} \sum_{y=1}^{N} A_{x y} \frac{k_{y}}{k_{y}+1}+r \sum_{y=1}^{N} A_{x y} \frac{1}{\left(k_{y}+1\right)^{2}}-\frac{k_{x}}{k_{x}+1}\right. \\
& \left.+\frac{r k_{x}}{k_{x}+1} \frac{\left\langle\frac{k}{k+1}\right\rangle}{\langle k\rangle}+\frac{r}{\left(k_{x}+1\right)^{2}}-\frac{1}{k_{x}+1}\right] \\
= & \rho_{c}\left[\frac{r}{\langle k\rangle^{2}}\left\langle\frac{k}{k+1}\right\rangle\left\langle\frac{k^{2}}{k+1}\right\rangle_{x}+\frac{r}{\langle k\rangle}\left\langle\frac{k}{(k+1)^{2}}\right\rangle k_{x}\right. \\
& \left.+\frac{r}{\langle k\rangle}\left\langle\frac{k}{k+1}\right\rangle \frac{k_{x}}{k_{x}+1}+\frac{r}{\left(k_{x}+1\right)^{2}}-1\right],
\end{aligned}
$$

where the numerically observed dependence of $\rho_{c}$ on the selection parameter $\alpha$ has been used. Predictions from Eq. (9) for the different values of $\alpha$ in Fig. 3 are shown as solid curves. We observe a good agreement with the numerical data. The linear degree-payoff relation can be obtained by setting $k_{x} \gg 1$ in Eq. (9). We obtain

$$
\begin{aligned}
M_{x} \approx & \rho_{c}\left[\frac{r}{\langle k\rangle^{2}}\left\langle\frac{k}{k+1}\right\rangle\left\langle\frac{k^{2}}{k+1}\right\rangle k_{x}+\frac{r}{\langle k\rangle}\left\langle\frac{k}{(k+1)^{2}}\right\rangle k_{x}\right. \\
& \left.+\frac{r}{\langle k\rangle}\left\langle\frac{k}{k+1}\right\rangle-1\right] .
\end{aligned}
$$

\section{B. Distribution of payoff}

Based on Eq. (9), we can derive the distribution of payoff, denoted by $P(M)$. It is also named wealth distribution in economics where an individual's wealth is determined by payoff. In general, we have

$$
P\left(k_{x}\right) d k_{x}=P(M) d M,
$$

where $P\left(k_{x}\right)$ is the node degree distribution. For the standard scale-free network, we have $P\left(k_{x}\right)=2 k_{\min }^{2} k_{x}^{-\gamma}$ with $\gamma=3$. From Eq. (9), we have

$$
\begin{aligned}
\frac{d k_{x}}{d M}= & {\left[\frac{\rho_{c} r}{\langle k\rangle^{2}}\left\langle\frac{k}{k+1}\right\rangle\left\langle\frac{k^{2}}{k+1}\right\rangle+\frac{\rho_{c} r}{\langle k\rangle}\left\langle\frac{k}{\left(k_{x}+1\right)^{2}}\right\rangle\right.} \\
& \left.-\frac{\rho_{c} r}{(k+1)^{2}\langle k\rangle}\left\langle\frac{k}{k+1}\right\rangle-\frac{2 \rho_{c} r}{\left(k_{x}+1\right)^{3}}\right]^{-1} .
\end{aligned}
$$

The wealth distribution can then be obtained as

$$
P(M)=P\left(k_{x}\right) \frac{d k_{x}}{d M}=G_{0}\left(k_{x}\right)\left[k_{x}(M)\right]^{-\gamma} .
$$

In general, the coefficient $G_{0}\left(k_{x}\right)$ depends on $k_{x}$ and it is given by

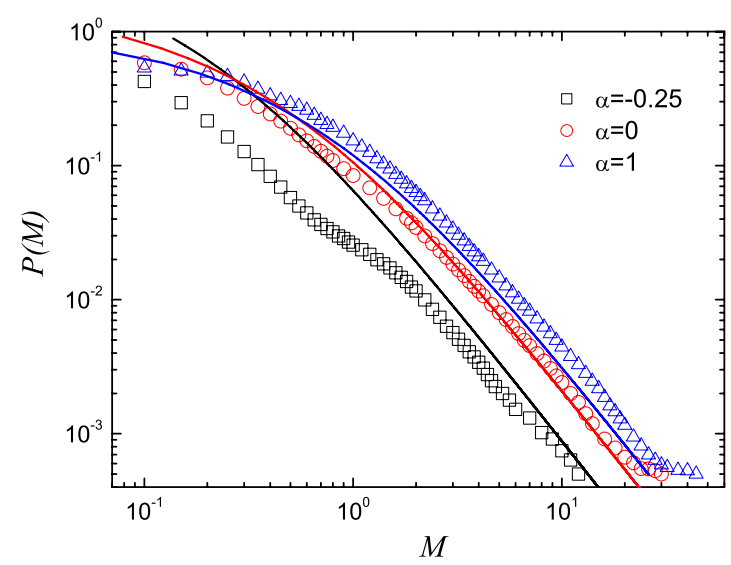

FIG. 4. (Color online) Cumulative payoff distribution for different values of $\alpha$. The distribution is obtained after the cooperation density becomes stable. The multiplication factor is set to be $r$ =1.6. Solid curves are theoretical predictions from Eq. (13).

$$
\begin{aligned}
G_{0}\left(k_{x}\right)= & k_{\min }\left[\frac{\rho_{c} r}{\langle k\rangle^{2}}\left\langle\frac{k}{k+1}\right\rangle\left\langle\frac{k^{2}}{k+1}\right\rangle+\frac{\rho_{c} r}{\langle k\rangle}\left\langle\frac{k}{(k+1)^{2}}\right\rangle\right. \\
& \left.-\frac{\rho_{c} r}{\left(k_{x}+1\right)^{2}\langle k\rangle}\left\langle\frac{k}{k+1}\right\rangle-\frac{2 \rho_{c} r}{\left(k_{x}+1\right)^{3}}\right]^{-1} .
\end{aligned}
$$

For high-degree nodes, $G_{0}$ is independent of $k_{x}$ and it is given by

$$
G_{0} \approx k_{\min }\left[\frac{\rho_{c} r}{\langle k\rangle^{2}}\left\langle\frac{k}{k+1}\right\rangle\left\langle\frac{k^{2}}{k+1}\right\rangle+\frac{\rho_{c} r}{\langle k\rangle}\left\langle\frac{k}{(k+1)^{2}}\right\rangle\right]^{-1} .
$$

Simulation results for the wealth distribution are shown in Fig. 4, which are consistent with the theoretical analysis. We note that the "fat-tail" power-law wealth distribution obtained here reproduces the empirical data from financial market [35] fairly well.

\section{Occurrence of optimal cooperation}

First, for $\alpha<0$, low-degree individuals have a larger probability to be selected to refer to. When $r$ is small, e.g., $r<3$ (the smallest group size in our present model), even the smallest group does not favor cooperation. In this case, learning from low-degree individuals who occupy most nodes in the network cannot promote cooperation. Second, note that, since hubs usually have much higher payoffs, their strategies will dominate their neighbors. As a result, for intermediate values of $\alpha$ (say, near the optimal value), lowdegree individuals around the defector hub (D-hub) would prefer to select D-hubs as references. As a result, the number of cooperators around a D-hub decreases rapidly, reducing quickly payoff for the hub. The payoffs of C-hubs can also decrease, but the decreases are typically insignificant. This is so because on a scale-free network, hubs share few neighbors so that most of the C-hub neighbors tend to follow the C-strategy. As a result, D-hubs become vulnerable to cooperators. Once an original D-hub is occupied by a cooperator, its neighbors will subsequently take the strategy of coopera- 


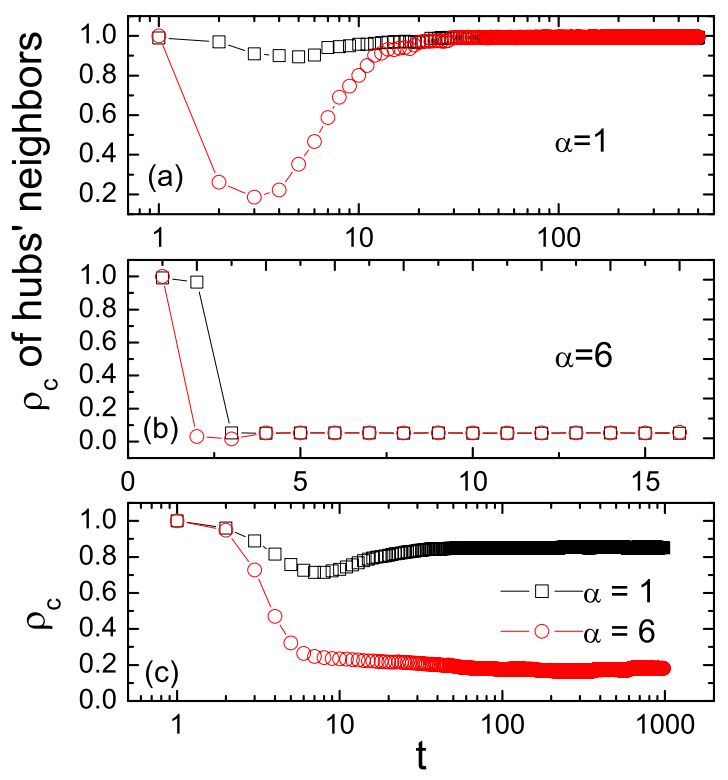

FIG. 5. (Color online) Times series of cooperator density in hubs' neighborhoods for (a) $\alpha=1$ and (b) $\alpha=6$. (c) Time series of cooperator density $\rho_{c}$ for the entire network for $\alpha=1$ and $\alpha=6$. The network size is 2000 and the average connectivity is $\langle k\rangle=4$. For (a) and (b), open circles and open squares indicate evolutions of cooperators around the highest-degree and the second highest-degree node, respectively, where the two hubs are connected and share five neighbors. Initially only the highest-degree individual is a defector and others are cooperators. The multiplication factor is $r=1.2$ and each data point is obtained by averaging over 50 runs.

tion, prohibiting the diffusion of defection. Third, for large values of $\alpha$, the probabilities for some hubs to be selected as references are also large. As a result, they pass their strategies to the majority of nodes that can act only as receivers. Exchanges of strategies among hubs are thus intensified, giving D-hubs better chances to survive. Note that the importance of information exchange among hubs for the emergence and persistence of cooperation has already been investigated in Refs. [36,37]. Combining all three regimes of $\alpha$, we can expect the existence of an optimal value of $\alpha$ that leads to a maximum degree of cooperation.

To provide support for the above scenario, we construct a special scale-free network where the highest-degree node has 103 connections and the second highest-degree node has 99 connections, and the two hub nodes are connected. Initially, only the highest-degree individual is a defector and all the other individuals are cooperators. We then compute the cooperator densities in the neighborhoods of the two hubs as functions of time, as shown in Figs. 5(a)-5(c). From Fig. 5(a), we see that, for $\alpha=1$, the cooperative neighbors around the C-hub only decrease by about $10 \%$ under the influence of the D-hub, and the cooperation density in the D-hub's neighborhood decreases rapidly initially and then increases to unity. At the turning point, the D-hub is occupied by a cooperator. For $\alpha=6$ [Fig. 5(b)], the cooperator density in the C-hub's neighborhoods remains high at the initial time step, but after the second time step, the number of cooperators around the $\mathrm{C}$-hub decreases rapidly, indicating that a defector has taken over the C-hub. Figure 5(c) shows the evolution of

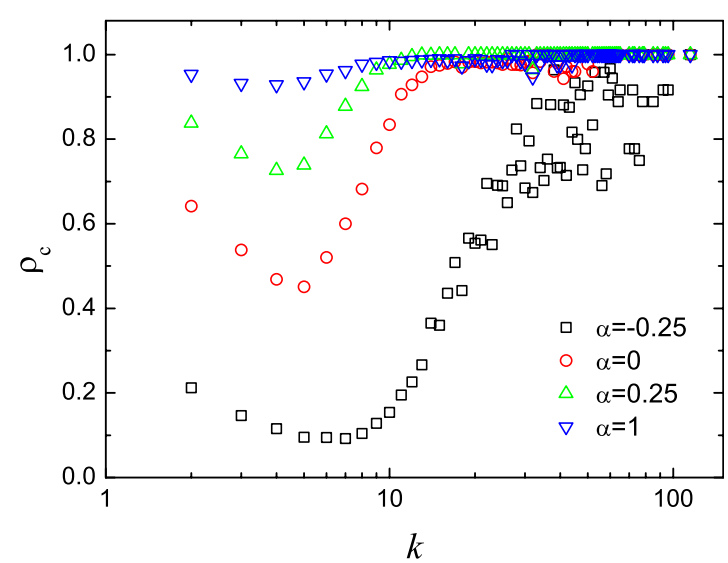

FIG. 6. (Color online) For $r=1.6$, cooperator density $\rho_{c}$ as a function of degree for different values of $\alpha$.

the cooperator density $\rho_{c}$ for the entire network for $\alpha=1$ and $\alpha=6$, where the initial condition is that there is a single defector at the highest-degree node. One can see that for $\alpha=1$, $\rho_{c}$ decreases at the beginning and then increases to a high value. For $\alpha=6, \rho_{c}$ decreases to a low value.

These results demonstrate the importance of information exchange between the two hubs and how the C-hub prevents the propagation of the defection strategy from the D-hub. For $\alpha=6$, because of the high weight of the D-hub, nearly all the neighbors of the D-hub choose it as a reference, including the C-hub with the second largest degree. Due to the higher payoff of the D-hub, all its neighbors become defectors at the second step, as shown in Fig. 5(b). At the next step, all cooperating neighbors of the hub with the second largest degree become defectors and finally defection spreads to nearly the entire network. This indicates that strong influence of hubs can facilitate defection. For $\alpha=1$, since the D-hub's weight is not so strong, it is not chosen by the C-hub despite that a large part of neighbors imitate the D-hub. As a result, the cooperator density around the D-hub decreases to about 0.3 , but $\rho_{c}$ around the C-hub changes a little, as shown in Fig. 5(a). At the next step, the D-hub's payoff is considerably reduced due to the death of many of its neighboring cooperators. Once the D-hub selects the C-hub, the D-hub will definitely change to be a cooperator and the spread of defection is prevented. The neighbors of the largest-degree hub finally become cooperators again, as shown in Fig. 5(c). From this analysis, we can infer that appropriate weights of hubs can induce optimal cooperation. Indeed, our simulation results show that the optimal value of the weight parameter $\alpha$ is about 1 and depends weakly on the value of $r$.

Figure 6 shows, for different values of $\alpha$, the density $\rho_{c}$ versus the degree variable $k$. We see that both high-degree and very low-degree nodes tend to possess high cooperation density, while the nodes with medium degrees are more likely to be defectors. As we have discussed, for intermediate values of $\alpha$, the $\mathrm{C}$-strategy is more stable for hub nodes than the D-strategy so that cooperators dominate high-degree nodes, as displayed in Fig. 6. For a scale-free network, lowdegree nodes are the majority and they are usually neighbors of high-degree nodes. These nodes will then be influenced by hubs and imitate their C strategy. As a result, very low- 


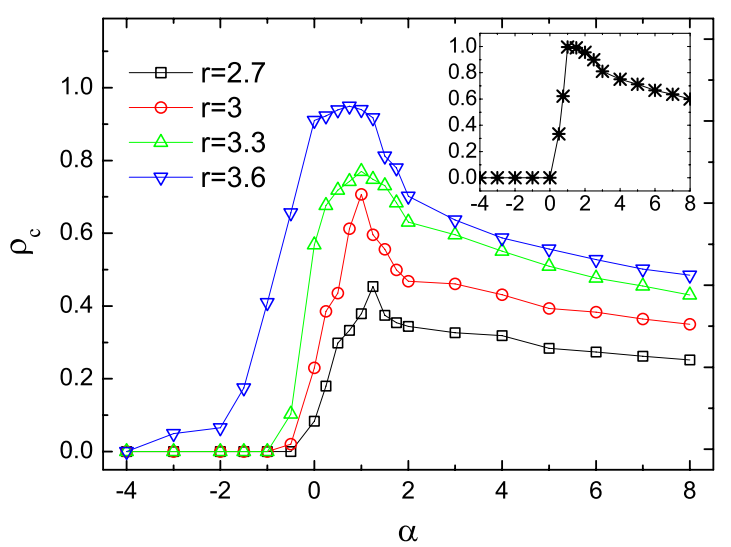

FIG. 7. (Color online) For $N=2000$ and $\langle k\rangle=4$, the density $\rho_{c}$ as a function of $\alpha$ for different values of $r$ in the case where all cooperators contribute the same cost $c$ per game. Every cooperator contributes a cost $c=1$ in every neighborhood that it plays. The inset is $\rho_{c}$ versus $\alpha$ for $r=4$ by using normalized payoffs.

degree nodes can also present relatively high cooperation density. For the medium degree nodes, they can gain more payoffs from their low-degree cooperator neighbors if they adopt the D-strategy. As a result, they are occupied by more defectors.

In the above analysis, we assume that each cooperator contributes the same total cost $c=1$, equally shared among all the neighborhoods that it engages, such that the individual contribution of each cooperator is independent of the number of social ties [13]. In the opposite limit considered in Ref. [13], every cooperator contributes a cost $c=1$ in every neighborhood that it engages. In this case, the total contribution of each cooperator $i$ is equal to $k_{i}+1$, effectively the number of its social ties. While we have found optimal cooperation in the PGG in the former limit, a question is whether the same occurs in the latter case. An affirmative answer is provided in Fig. 7. Cooperation depending $\alpha$ for the case of normalized is studied as well, as shown in the inset of Fig. 7. The existence of optimal cooperation induced by preferential imitation is thus a robust phenomenon, regardless of whether the contribution of cooperators is related to the number of their social ties and whether payoffs are normalized [38].

\section{CONCLUSION AND DISCUSSIONS}

We have developed a framework to investigate the phenomenon of diversity-promoted cooperation in evolutionary game theory. This is motivated by the previous qualitative finding that cooperation among individuals can be enhanced by diversity among them. The phenomenon is somewhat counterintuitive because naively, one would expect cooperation to be favored among individuals of similar characteristics. However, it is intuitively reasonable that cooperation would not benefit if diversity is too strong. To resolve this dilemma, it is necessary to be able study the interplay between diversity and cooperation in a quantitative manner.

To quantify the degree of diversity, we take advantage of complex networks and tacitly assume that all individuals (game players) are connected with each other, exhibiting some heterogeneous connectivity (degree) distribution. The setting is in fact quite general as cooperation typically occurs on networked systems. To be able to systematically assess the effect of diversity on cooperation, we have introduced a diversity parameter in strategy updating. We have found that a maximal degree of cooperation can be achieved for an optimal level of diversity. The key to understanding this resonancelike phenomenon lies in the role played by highly connected individuals, or hubs, in the game. In particular, while hubs are usually occupied by cooperators for moderate level of diversity, which leads to strong cooperation, we find that defectors can take over the hubs if the individual game players are too diverse. We have also addressed the related issue of payoff distribution and obtained a theoretical prediction that is matched by realistic distributions. Evolutionary games are an important tool for understanding a large array of phenomena in complex systems, and our results have provided new insights into the role played by diversity in promoting cooperation.

\section{ACKNOWLEDGMENTS}

This work is funded by the National Basic Research Program of China (973 Program No. 2006CB705500), the National Natural Science Foundation of China (Grants No. 60744003 and No. 10635040£10532060), the Specialized Research Fund for the Doctoral Program of Higher Education of China (Grant No. 20060358065), and National science fund for fostering talents in basic science (Grant No. J0630319). W.X.W. and Y.C.L. are supported by AFOSR (Grant No. FA9550-07-1-0045). Z.X.W. is supported by the Swedish Research Council (Project No. 540042101).
[1] A. M. Colman, Game Theory and Its Applications in the Social and Biological Sciences (Butterworth-Heinemann, Oxford, 1995).

[2] J. M. Smith, Evolution and the Theory of Games (Cambridge University Press, Cambridge, England, 1982).

[3] H. Gintis, Game Theory Evolving (Princeton University Press, Princeton, NJ, 2000).

[4] R. Axelrod and W. D. Hamilton, Science 211, 1390 (1981).

[5] C. Hauert and M. Doebeli, Nature (London) 428, 643 (2004);
M. Sysi-Aho, J. Saramäki, J. Kertész, and K. Kaski, Eur. Phys. J. B 44, 129 (2005); L.-X. Zhong, D.-F. Zheng, B. Zheng, C. $\mathrm{Xu}$, and P. M. Hui, Europhys. Lett. 76, 724 (2006); A. Szolnoki and G. Szabó, EPL 77, 30004 (2007); G. Abramson and M. Kuperman, Phys. Rev. E 63, 030901(R) (2001); B. J. Kim, A. Trusina, P. Holme, P. Minnhagen, J. S. Chung, and M. Y. Choi, ibid. 66, 021907 (2002); H. Ebel and S. Bornholdt, ibid. 66, 056118 (2002); Z.-X. Wu, X.-J. Xu, Y. Chen, and Y.-H. Wang, ibid. 71, 037103 (2005); W.-X. Wang, J. Ren, G. Chen, 
and B.-H. Wang, ibid. 74, 056113 (2006); C.-L. Tang, W.-X. Wang, X. Wu, and B.-H. Wang, Eur. Phys. J. B 53, 411 (2006); J. Ren, W.-X. Wang, and F. Qi, Phys. Rev. E 75, 045101(R) (2007); A. Szolnoki and G. Szabo, EPL 77, 30004 (2007); X. Chen and L. Wang, ibid. 77, 017103 (2008); F. Fu and L. Wang, ibid. 78, 016104 (2008); F. Fu, C. Hauert, M. A. Nowak, and L. Wang, ibid. 78, 026117 (2008); W.-X. Wang, J. Lü, G. Chen, and P. M. Hui, ibid. 77, 046109 (2008).

[6] J. H. Kagel and A. E. Roth, The Handbook of Experimental Economics (Princeton University Press, Princeton, NJ, 1997).

[7] C. Hauert, S. De Monte, J. Hofbauer, and K. Sigmund, Science 296, 1129 (2002).

[8] G. Szabó and C. Hauert, Phys. Rev. Lett. 89, 118101 (2002).

[9] C. Hauert, S. De Monte, J. Hofbauer, and K. Sigmund, J. Theor. Biol. 218, 187 (2002).

[10] D. Semmann, H. J. Krambeck, and M. Milinski, Nature (London) 425, 390 (2003).

[11] H. Brandt, C. Hauert, and K. Sigmund, Proc. Natl. Acad. Sci. U.S.A. 103, 495 (2006).

[12] J. Y. Guan, Z. X. Wu, and Y. H. Wang, Phys. Rev. E 76, 056101 (2007).

[13] F. C. Santos, M. D. Santos, and J. M. Pacheco, Nature (London) 454, 213 (2008).

[14] H. Ohtsuki and Y. Iwasa, J. Theor. Biol. 231, 107 (2004).

[15] M. A. Nowak and K. Sigmund, Nature (London) 437, 1291 (2005).

[16] C. Hauert, A. Traulsen, H. Brandt, M. A. Nowak, and K. Sigmund, Science 316, 1905 (2007).

[17] See, for example, K. Wiesenfeld and F. Moss, Nature (London) 373, 33 (1995); L. Gammaitoni, P. Hänggi, P. Jung, and F. Marchesoni, Rev. Mod. Phys. 70, 223 (1998).

[18] G. Szabó and C. Töke, Phys. Rev. E 58, 69 (1998); G. Szabó and C. Hauert, Phys. Rev. Lett. 89, 118101 (2002); G. Szabó and J. Vukov, Phys. Rev. E 69, 036107 (2004).

[19] M. Perc and A. Szolnoki, Phys. Rev. E 77, 011904 (2008).

[20] Z. X. Wu, X. J. Xu, Z. G. Huang, S. J. Wang, and Y. H. Wang, Phys. Rev. E 74, 021107 (2006).

[21] J. Y. Guan, Z. X. Wu, Z. G. Huang, X. J. Xu, and Y. H. Wang, Europhys. Lett. 76, 1214 (2006).

[22] J. Ren, W. X. Wang, G. Yan, and B. H. Wang, e-print arXiv:physics/0603007.

[23] A. Szolnoki and G. Szabó, EPL 77, 30004 (2007).

[24] A. Szolnoki, M. Perc, and G. Szabó, Eur. Phys. J. B 61, 505 (2008).

[25] G. Szabó and J. Vukov, Phys. Rev. E 69, 036107 (2004).

[26] G. Szabó, J. Vukov, and A. Szolnoki, Phys. Rev. E 72, 047107 (2005).

[27] M. Perc, Phys. Rev. E 75, 022101 (2007).

[28] Z. X. Wu and Y. H. Wang, Phys. Rev. E 75, 041114 (2007).

[29] A. L. Barabási and R. Albert, Science 286, 509 (1999).

[30] G. Szabó and G. Fáth, Phys. Rep. 446, 97 (2007).

[31] C. Hauert and G. Szabó, Complexity 8, 31 (2003).

[32] C. Hauert, Adv. Complex Syst. 9, 315 (2006).

[33] F. C. Santos and J. M. Pacheco, Phys. Rev. Lett. 95, 098104 (2005).

[34] F. C. Santos, J. M. Pacheco, and T. Lenaerts, Proc. Natl. Acad. Sci. U.S.A. 103, 3490 (2006).

[35] V. Pareto, Le Cours d'Économie Politique (Macmillan, Lausanne, Paris, 1987).

[36] Z. Rong, X. Li, and X. Wang, Phys. Rev. E 76, 027101 (2007).

[37] M. Perc, A. Szolnoki, and G. Szabó, Phys. Rev. E 78, 066101 (2008).

[38] We have also studied the prisoner's dilemma game on scalefree networks and found the phenomenon of diversityoptimized cooperation. 